\title{
Improving Airport Performance through a Model-based analysis and optimization approach
}

\author{
M. Mujica $\operatorname{Mota}^{(a)}$, P. Scala ${ }^{(b)}$, D. Delahaye ${ }^{(c)}$ \\ ${ }^{(a, b)}$ Aviation Academy, Amsterdam University of Applied Sciences, The Netherlands

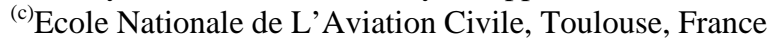

\begin{abstract}
Traditionally airport systems have been studied using an approach in which the different elements of the system are studied independently. Until recently scientific community has put attention in developing models and techniques that study the system using holistic approaches for understanding cause and effect relationships of the integral system. This chapter presents a case of an airport in which the authors have implemented an approach for improving the turnaround time of the operation. The novelty of the approach is that it uses a combination of simulation, parameter analysis and optimization for getting to the best amount of vehicles that minimize the turnaround time of the airport under study. In addition, the simulation model is such that it includes the most important elements within the aviation system, such as terminal manoeuvring area, runway, taxi networks, and ground handling operation. The results show clearly that the approach is suitable for a complex system in which the amount of variables makes it intractable for getting good solutions in reasonable time.
\end{abstract}

\section{Introduction}

Air global transportation is in continuous growth, looking at the most recent statistics European flights have increased by $0.7 \%$ in May 2015 compared with the same month of the last year and it was above the forecast, furthermore preliminary data for June 2015 say that there will be a $1.2 \%$ of flights increase compare to June 2014 [10]. The majority of nations in Europe have seen a growth in their local flight, there are reports that mention the levels of congestion the airports in Europe are facing $[10,12]$. The direct effect of congestion in the airports is delays that correlate with the increasing traffic. The numbers of EUROCONTROL (the European organization for the safety or air navigation) [11] illustrate how the percentage of delayed flight in December 2016 increased by approximately 7\% when compared to the same month in the previous year. These situations make evident that capacity in airports is being chocked with the increase on traffic, and this situation might become dramatic if the forecasts of Boeing and EUROCONTROL are correct [10]. For this reason scientific community has paid 
a lot of efforts for developing tools, new paradigms and novel infrastructure that alleviates the different congestion problems that arise when the traffic increases. These solutions range from optimization tools, re-allocation paradigms or the design of novel infrastructures that have flexibility among their characteristics [8].

\subsection{Case Study: Lelystad Airport}

Amsterdam Schiphol (AMS) is the main airport in the Netherlands and it was the fifth busiest airport in Europe in 2014 in terms of passenger traffic [1]. Furthermore AMS is also the main hub for KLM, which provided 54\% of the seats available at the airport in 2013, and a major airport for the SkyTeam alliance, whose members - including KLM - are responsible for $66.3 \%$ of the airport traffic in terms of ATM [27]. Its role as a hub, by airport management and government, is central to the airport strategy, especially considering the small size of the domestic market in the Netherlands and the airport's role as economic engine for the region. However due to environmental reasons, the capacity is limited to 510,000 air traffic movements per year (landings and departures). In 2015 there were 450,679 movements at the airport, $91 \%$ of the imposed cap [29]. Since the operation is approaching to the limits, Schiphol Group would like to support the airport strategy by redistributing traffic non-related to the hub development to other airports in the Netherlands. The objective of this action is to relieve capacity and at the same time continuing providing support for the development of the region. The preferred alternative is to upgrade Lelystad Airport (LEY) to attract commercial flights of European cities and regions [28], putting focus on tourist destinations. In that way LEY will take an important role in the multi airport system of the Netherlands composed currently by Schiphol, Rotterdam and Eindhoven.

In recent years Low Cost Carriers (LCCs) in Europe have put focus on shorthaul point-to-point leisure traffic, in addition they have been targeting business travellers more actively, and some of them even offer interline connectivity using simple hub structures. This means that the development process at Lelystad should consider not only the type of passengers and airlines that are desired but also the performance parameters the airport should have in order to become attractive for these types of carriers since the airports cannot force the activity in it, instead they make the airport attractive through the offering of incentives economical and operative ones.

Lelystad is the largest airport for general aviation traffic in the Netherlands. It is located $56 \mathrm{~km}$ from central Amsterdam, about 45 minutes by car to the east. The airport is fully owned by the Schiphol Group, which also owns Rotterdam airport (RTM) and a 51\% stake in the Eindhoven airport (EIN), both in the Amsterdam Multi-Airport System (see Figure 1). 


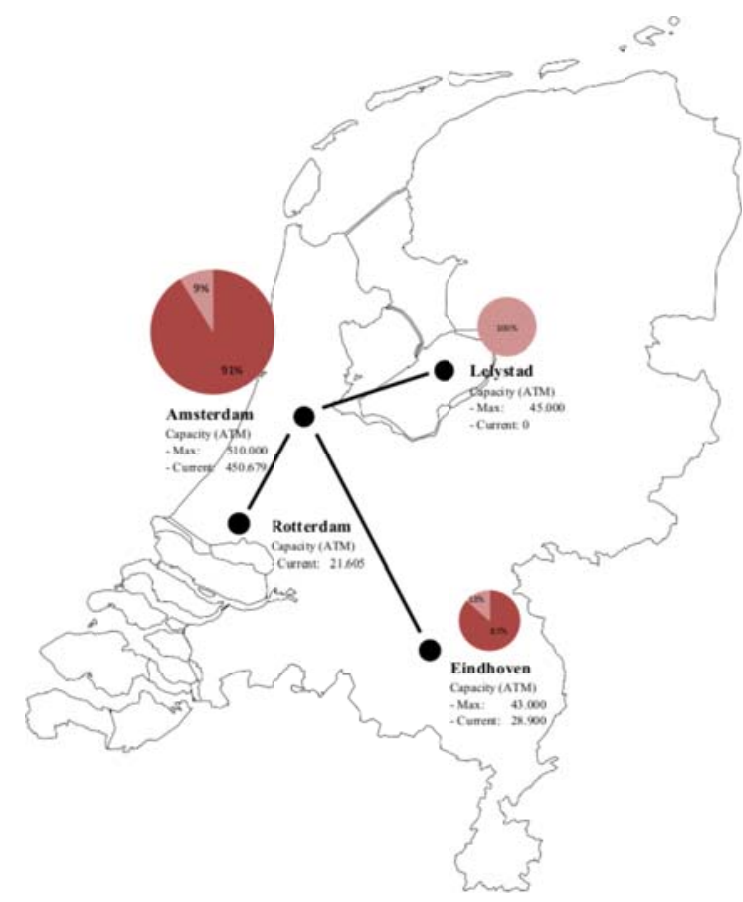

Fig. 1 Lelystad and the multi airport system of the Netherlands

In order to attract airlines, especially LCCs, Lelystad would need to provide differentiation factors: availability of slots; low aeronautical charges; incentive programs and quick aircraft turnaround [15]. Therefore, in order to have better insight about the operational performance of the future airport it is necessary to have tools that provide information about the future performance of it and powerful enough that allow identifying the emergent dynamics once the operation is in place.

Traditional analytical tools fall short on the capacity to understand the operative problems that a future facility will face. Nowadays only model-based tools appear as the ones with the capacity and analytical power for integrating the different restrictions and factors that influence the performance of the systems while taking into account the inherent stochasticity present in the systems. For these reasons we present a model-based approach in which the simulation model was developed based on public information for identifying the optimal performance of it. 
Designing the layout and the infrastructure of an airport means allocating resources in a way that traffic matches demand without incurring in congestion situations. Furthermore, since the objective of the airport operator aims also at minimizing the size so that the final infrastructure is not half empty when the time comes the right balance must be found. In this context, evaluating the performance of the airport has become a crucial aspect, especially if the aim is to efficiently manage the existing resources.

Regarding the quality of service, from the passenger perspective, the main factor that affects quality is the delay, so the less delays; the more positive evaluations can be obtained from passengers. From the airport perspective, having less delays can lead to more capacity for processing more aircraft, hence an increment in capacity. This in turn leads to have higher revenues and the opportunity to attract more airlines since more slots could be allocated. Additionally the better level of service will cause the increment in passengers choosing that airport as origin and destination.

The airport system is composed by different elements, the terminal area, the airside (runway, taxiways and stands) and then the airspace (sectors, routes, terminal manoeuvring area). These components are often analysed separately, but in reality these components are tied to each other, and they act all together as a system in which the good or bad performance in one element affects the others. Motivated by this fact, in this work it was made an analysis of the performance of an airport system, taking into account all the components previously mentioned.

The methodology applied in this work is a combination of simulation and optimization that takes into account all the components of an airport system (ground + airspace), and evaluates the airport performance in terms of the turnaround time (TAT). The simulation paradigm used in this work is a Discreteevent Simulation (DES) in a program called SIMIO [31]. The optimization approach is a simulation-based optimization in which the search space is the domain of the Cartesian product of the values of the main factors that affect the objective function; the search is performed by an embedded tool called OptQuest [23] that has different heuristics for optimizing the search.

With the use of the simulation model, different configurations of resources were evaluated paying attention to the TAT. The use of design of experiments (DOE) was carried out employing a multi-level factorial design with the purpose of evaluating the effect of the factors and their interactions for the system response. Moreover, with the study of the Analysis of Variance (ANOVA), the main factors that affect the objective function were determined. Finally, for optimizing the TAT, we used the information of the identified factors for making the optimization search more efficient than the one that could be done without the analysis. 


\subsection{Previous Work}

Optimization of airport resources is a subject that was faced by researchers in many studies; most of them treated the airport as a two separate entities, from one side airspace and from the other ground side. In this context, many techniques that aimed at improving airport performance were employed, taking into account different variables. Concerning the airspace, specifically for the Terminal Manoeuvring area (TMA) many studies focused on the sequencing and merging problem and scheduling problem. The former is concerned in finding the best sequence for aircraft flow in order to determine conflict-free situations[18, 33, 34], the latter is about scheduling of aircraft flow in order to minimize the deviation between the scheduled landing time and the actual landing time [4, 5, 6, 3, 21].

The techniques most utilized were from the operations research arena in which some of the solutions used stochastic optimization models [2], however, due to the complexity of the problems, for many of them heuristics were implemented in order to find sub optimal solutions. Just to mention some, the aircraft scheduling problem was studied extensively by Beasley et al. $[4,5,6]$ this work focused on developing a mixed-integer one-zero problem and then the authors employed two heuristics respectively for the static and dynamic case. Other relevant work is the one from Balakrishnan at al. [3], which uses constrained position shifted (CPS) for improving the sequence of aircraft by changing the position of the aircraft in order to minimize the make span. Hu and Chen [13] proposed a receding horizon control (RHC) technique where the scheduling and sequencing problem were treated in a dynamic way; they introduced a genetic algorithm for solving it.

Regarding the ground side, most of the studies are related to the optimization of gate assignment, the scheduling of departing aircraft and taxiing operations, with the objective of avoiding congestion situations and favouring a smooth flow of aircraft in the taxiways. For instance, in the work of Dorndorf [9] the authors present a survey about the techniques used to cope with the gate assignment problem, among others we can find the work of Bolat [7] in which a branch and bound algorithm was combined with two heuristics. A Coloured petri net (CPN) technique was proposed by Narciso and Piera [22] in order to calculate the number of stands needed to absorb the traffic. In other studies pushback control strategies were proposed in order to determine the best sequence of departures without incurring in congestion situations [24, 30, 16].

As it can be seen for the previous review, the most implemented techniques refer to analytic and heuristic models, and there is a clear distinction between airspace and ground side. In this work the problem is treated from a holistic view in which both airspace and ground side are analysed together thus making a more complete study. Additionally, a methodology has been followed that permits optimizing airport performance following a structured way. The approach focuses in performance measured as turnaround time which is the key for determining the amount of resources an airport needs in order to improve throughput and reduce delays due to congestion. 
The chapter continues in the following way, in section 2 the methodology used is presented, in section 3 the results from the different design of experiments and from ANOVA are presented, finally in section 4 the correspondent conclusions are presented.

\section{Methodology}

The approach uses first Discrete Event Simulation (DES) together with statistical techniques for identifying the most influencing factors in the performance of the airport under study. After performing an analysis of the different factors that influence the performance, they are disaggregated for making a more refined selection of those. The identification of the ultimate ones allow for reducing the search space of the optimization tool embedded in the simulation program used.

DES is an approach that is used in many applications like logistic and manufacturing [17]. Recently DES was also applied to the aviation field with the scope of modelling the airport operation for both airspace and ground, even inside the terminal [19]. Using this approach, it has been possible to make an initial analysis and evaluation of airports performance [20, 25, 26]. The methodology uses statistical tools like Design of experiments and the ANOVA for identifying the factors that impact the system the most and a selection of parameters is done which at the final stage will be used to optimize the values of the most influential elements of the system.

The methodology applied works in phases, in the first phase it performs the identification of the factors that affect the performance of the airport using an objective function of the turnaround time. During this phase the significance of the different factors that affect such performance are identified and then a combination of DOE with ANOVA is performed for making a more refined selection of the elements that affect the indicator.

In a second phase the model is combined with an optimization algorithm for performing the improvement of the system under study in which the decision variables are the ones that affect the objective function.

Figure 2 illustrates the different phases of the methodology 


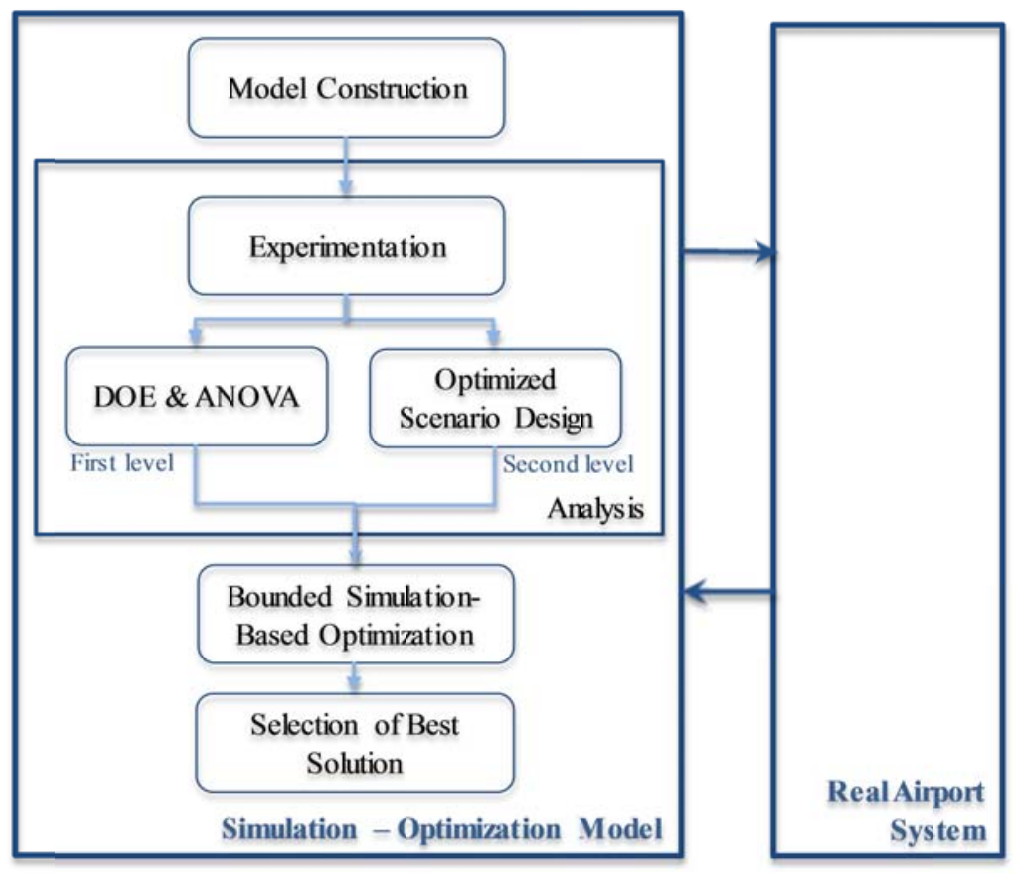

Fig. 2 Methodological approach for airport optimization

\subsection{Development of the airport model and Identification of Variables}

Until recently, scientific community has been taken simulation as a key tool for evaluating systems performance during the planning phase of facility development. In the aviation field the studies concerning systems performance and capacity evaluation are quite recent but its potential has been recognized by international institutions and also as consultants which are becoming keen for the use of simulation for performing studies [19, 25, 26].

The first phase focuses on the development of a simulation model of the system under study and the identification of the main variables. In the case of the airport of this work, we used the DES approach. This is an approach that is used for modelling systems of dynamic nature in which there is strong interaction between the different processes of the system and stochasticity is one of the characteristics that define them. In comparison with other approaches, the time advances as events are happening in the model, so the number of calculations is much less than the ones required for agent-based technology for instance. As most of the 
simulation approaches, it allows the identification of emergent dynamics within the system and it has the full potential for integrating the inherent stochasticity which in some situations hinders the smooth behaviour of the system under study. This approach has been successfully used in logistics and manufacturing systems for identifying emergent behaviour, the impact of variability and uncertainty and for bottleneck identification among other characteristics [20]. The tool we selected for the approach is SIMIO [31] which possesses the aforementioned properties besides an efficient management of graphical aspects and it takes also the advantages of the computer resources. In addition, it allows developing the models in a modular fashion which is very suitable for the study we are presenting.

The modular approach [26] allows putting focus only on one part of the model at the time and once it is verified, validated and finalized it can be coupled with other modules for obtaining the final one. The authors suggest strongly this approach since the reliability of the final model is higher if we perform a bottomup modelling approach. Figure 3 illustrates the proposed approach for this phase.

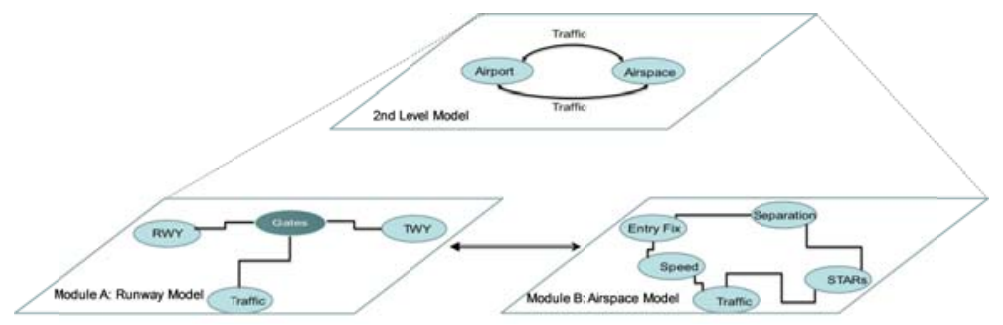

Fig. 3 The bottom-up modular modelling approach

For the developed model, the main components of airside and airspace were included like:

- Runway system

- Taxiway system and stands

- Approaching and departing routes

- Airspace

The architecture of the different modules' models is illustrated by Figure 4. 


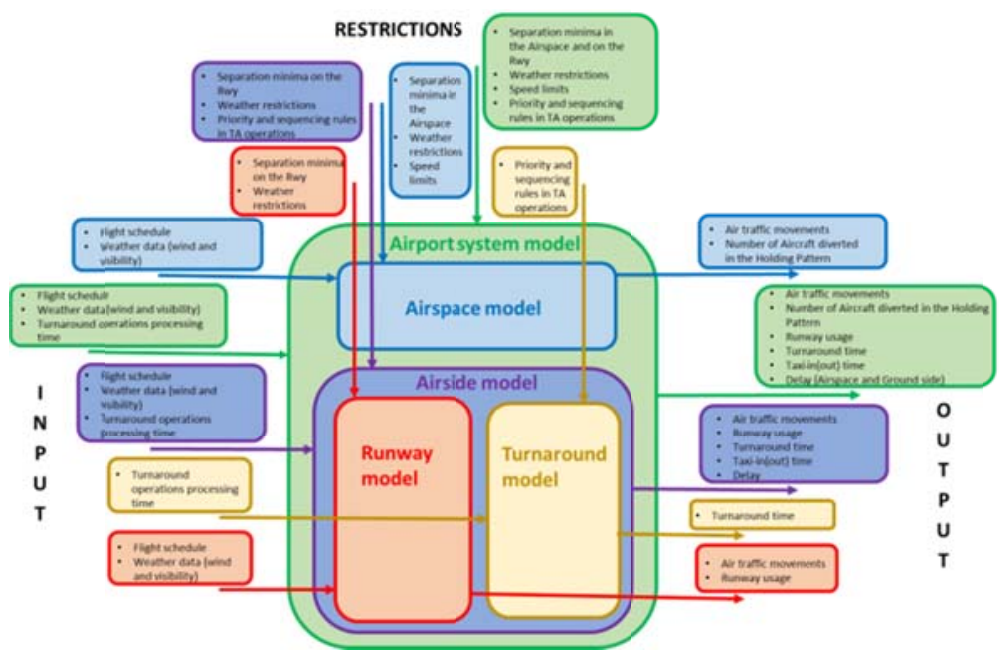

Fig. 4 Modular architecture of the Airport model

The airside is made by a coupling of two modules: the runway model and the turnaround model.

The runway model integrates the main characteristics and restrictions of the utilization of any runway in an airport system such as wake vortex separations, speed limitations, taxi speed and limitation of the runway.

The turnaround model is made by a model in which all the services required by an aircraft at the gate are implemented. These services are performed by a number of vehicles dedicated to providing them. Table 1 illustrates the different implementations and restrictions of the runway and turnaround model.

Table 1 Characteristics of the runway and turnaround model

\begin{tabular}{ll}
\hline Parameter & Value \\
\hline Number of runways & 1 \\
Number of exit ways & 1 \\
Taxiway type & Parallel \\
Number of stands & 16 \\
Aircraft speed Taxi in & {$[45$ Knot..24Knot] } \\
Aircraft speed Taxi out & [19 Knot] \\
\hline
\end{tabular}

The last module that composes the integral model is the one of the airspace close to the airport, in particular the area known as terminal manoeuvring area, which is composed by a radius of approximately 40 nautical miles. This area is important since it is within its limits that the sequencing of arrivals is performed. The modelling approach uses a network of nodes located at different altitudes and 
positions in the space in which the connections between them represent the airways followed by the aircraft in their routes to the airport. Due to the scale of the model, the airport itself just represents one segment of the network. Figure 5 illustrates the network created for modelling the airspace.

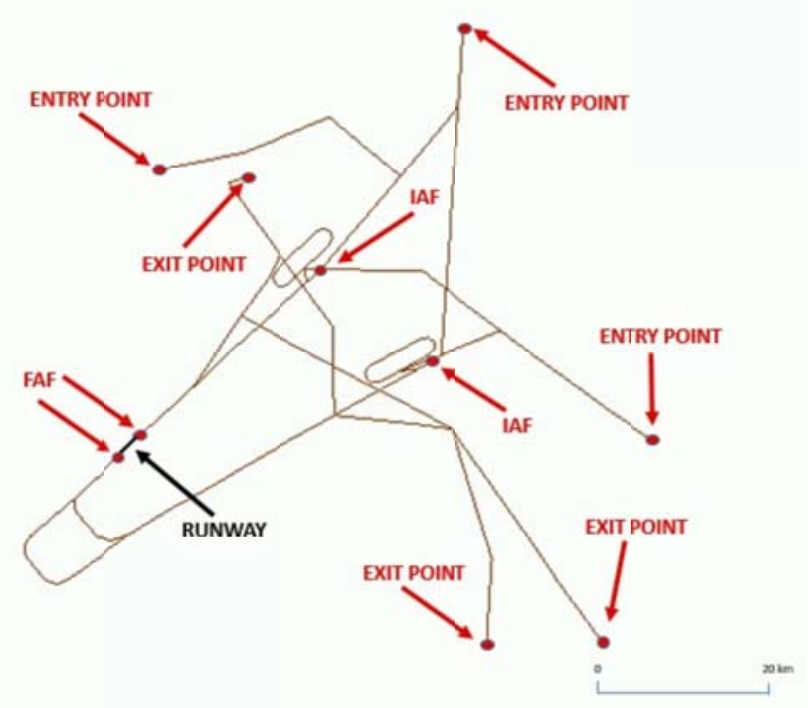

Fig. 5 TMA Airspace Network

The acronyms present in the figure refer to Initial Approach Fix (IAF) and Final Approach Fix (FAF) which are the segments in which the controllers guide the aircraft for their final route to landing [32]. The basic restrictions that must be taken care of for the development of the simulation model are the separations that need to be respected by the aircraft to land. These separations are for safety reasons which ensure the minimization of the risk of collision or interactions between the aircraft in the area surrounding the airport. 
Table 2 and Table 3 present the description of the different restrictions and parameters that compose the model.

Table 2 Characteristics of the TMA model

\begin{tabular}{llc}
\hline Parameter & Value & Value \\
\hline Entry point (Speed) & 250 Knot & 160 Knot \\
Initial approach fix (Speed) & 160 Knot & 130 Knot \\
Final approach fix (Speed) & - & 130 Knot \\
\hline Holding pattern & 1 for each route, 200 Knot & \\
(number and speed limit) & & \\
Aircraft mix & Code C (B737 - A320) \\
\hline
\end{tabular}

Table 3 Separation Minima in Nautical Miles (ICAO)

\begin{tabular}{llccc}
\hline & & \multicolumn{3}{c}{ Leading Aircraft } \\
\cline { 2 - 5 } Trailing aircraft & Heavy & Medium & Light \\
\cline { 2 - 5 } & Heavy & 4 & 3 & 3 \\
& Medium & 5 & 3 & 3 \\
& Light & 6 & 4 & 3 \\
\hline
\end{tabular}

Once the three models have been developed and validated against expected speeds and relevant variables they were merged into a one integral model that represents the airport system (airside and airspace). The different modules interact with each other in such a way that it is possible to evaluate the behaviour of different performance indicators (PI) and the emergent dynamics which would not be possible to perceive if the models were analysed independently. Figure 6 depicts the complete model, in which the entities first are generated in the airspace model, then they are sequenced for landing and the landing is performed. For the landing process they get out of the airspace model and enter the airside model. In the airside all the landing and taxiing is performed until the aircraft gets to the gate in which the turnaround operation is performed by the ground handling vehicles. 


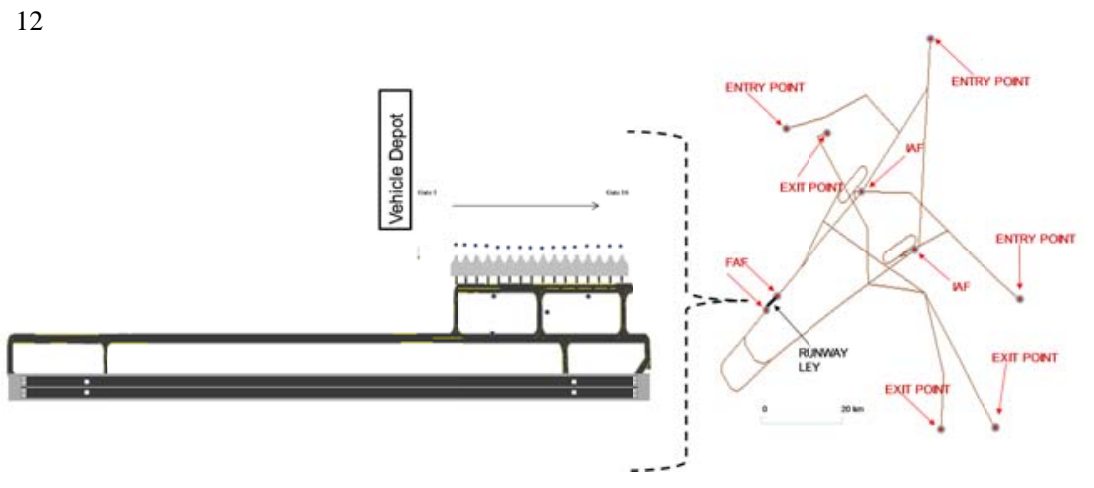

Fig. 6 Complete Model of the Airport of Lelystad

With the model developed, it was possible to evaluate performance indicators (PI) like number of movements, data about turnaround time and about delay under different scenarios. As an initial approach focus was put on the analysis of the TAT which is very important for understanding the potential of the airport under study.

\section{Getting more insight: Design of Experiments}

Design of experiments is a technique that permits to identify the main parameters, or factors that affect the performance of a system. With this technique, it is possible to evaluate what the main effects of the factors involved are, and also the effect of their interactions. This technique allows identifying the main effects for each factor. To that end, for each factor different values were assigned, called levels. For each combination of factor level a response is evaluated and an analysis is performed in order to identify if the factor is statistically significant for the studied variable.

This phase focuses on developing structured experiments with the model for identifying the most relevant factors.

For the example we present, we applied recurrently the technique in the simulation model to make an identification of the variables that affect the outcome of the PI the most. For the first and second level analysis we put the focus on the objective under study: Turnaround Time.

Turnaround time (TAT): This parameter is the time measured from the moment the aircraft parks in the stand until it is ready for taxing out to the runway. This is an absolute number, so if the operation is delayed due to the lack of any of the vehicles or resources, the TAT will be increased as well. This number is important to know since it provides the airlines information about how efficient an operation in a particular airport could be. 


\subsection{First level of Analysis}

As the reader might assume, there are multiple sources or variables that can impact the TAT. For instance, the amount of available gates, vehicles for performing the operation, traffic level and more. The first approach in trying to identify the most relevant ones we needed to apply DOE in categories that group some factors. This selection was based on expert opinion and the selected ones were: air traffic, available vehicles for the turnaround, and stand allocation. Using these factors we performed a multi-level full factorial design. Table 4 illustrates the different categories of factors we evaluated for the design.

Table 4 Evaluated Category Factors

\begin{tabular}{lccc}
\hline Factors & Level 1 & Level2 & Level 3 \\
\hline A - Incoming flow of aircraft (flights/day) & 92 & 132 & 190 \\
B - Number of vehicles & 2 & 5 & 8 \\
C - Apron's entering mode & Left-Right & Center - Out & -
\end{tabular}

For the first and the second factors we set three levels and two levels for the last one. In addition, 50 replications were made for each level.

The evaluated levels for the three factors followed the following logic:

- Incoming Flow of aircraft. As it has been mentioned, this study deals with the evaluation of a future airport in the Netherlands. The public information states that the amount of expected traffic is approximately 50,000 ATMs per year. Thus the Level 2 is approximately this value so this traffic is considered the one expected by the airport. The other two levels explored the situation in which $30 \%$ more and $30 \%$ less traffic than expected is received in the airport.

- Number of Vehicles. The number of vehicles refers to the sets of vehicles that can be used for the operation. Without economical limitations we can estimate that we might use one complete set per aircraft, thus the initial set is of 9 vehicles. One set itself is composed by 1 fuel,2 passenger bus, 1 water, 2 bulk trucks, 2 stair trucks and 1 loader. The other two levels are used for evaluating the reduction in vehicles so that it is possible to perceive when the turning point is (if there is) of performance due to the lack of vehicles.

- Apron's entering mode. For this factor, only two levels were evaluated, they concerned with how the aircraft were allocated in the available stands. The two levels are, from left to right, assuming a first-in first-served allocation putting priority in the stands closest to the left part of the apron and the center-right allocation. This allocation assumes that the priority is put in the central stands. 


\section{Analysis of Variance (ANOVA)}

Once we have run the full factorial analysis, we performed the one-way ANOVA for identifying the impact of the different categories evaluated. Figure 7 presents the results obtained with the ANOVA test.

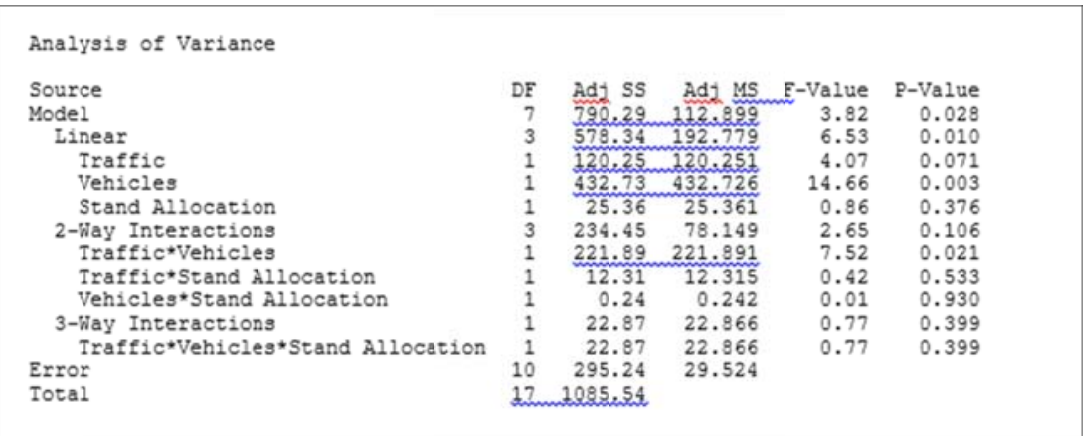

Fig. 7 Analysis of variance for the factors of TAT

From the ANOVA we could identify that the most significant category is the vehicles set since the $p$ value is smaller than 0.05 . This category is followed by traffic; however statistics cannot support conclusively this assumption. In addition we can also perceive that the interaction of traffic with vehicles is significant. So, as expected, traffic itself affects, however that is a variable in which we cannot manipulate to get a better or worse performance. For this reason we paid attention to the amount of vehicles in order to going further in the analysis.

Figure 8 depicts the Pareto chart of standardized effects to graphically illustrate the diverse effects of the different categories evaluated. 


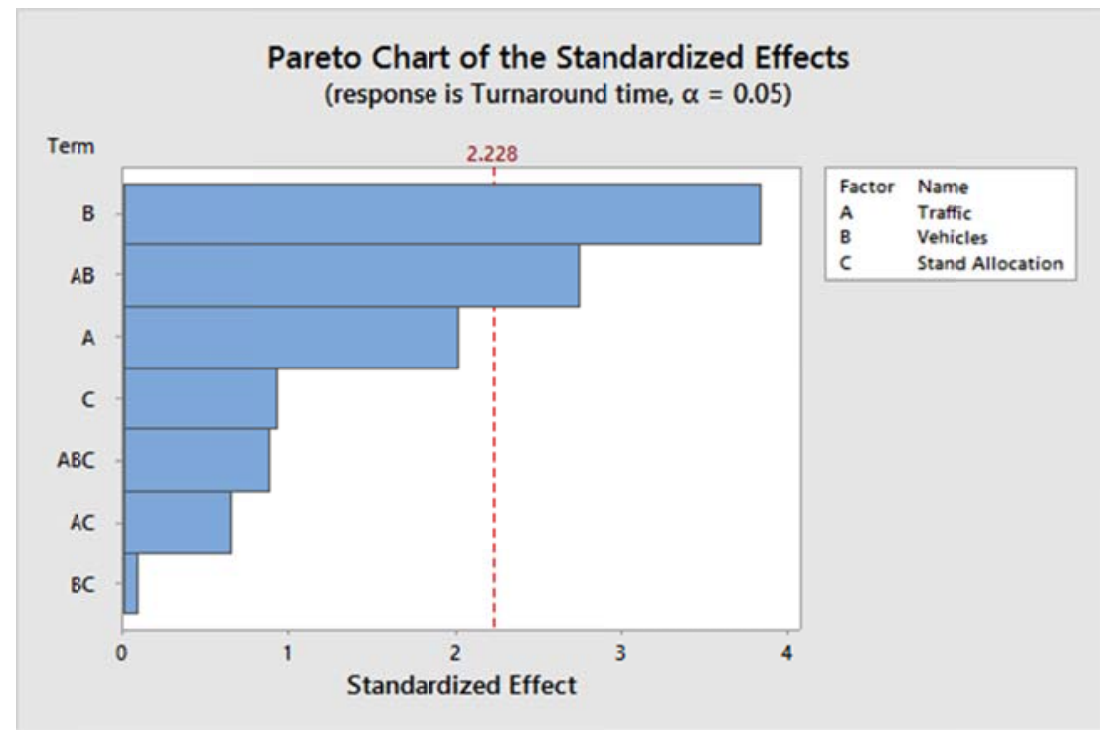

Fig. 8 Plot of the standardized effects for Turnaround time

Once we selected as vehicles as the most influential and controllable factor, the next question that arise is what the right mix of vehicles would be for a smooth and efficient operation.

\subsection{Second Level of Analysis}

For the turnaround objective, we run the second level DOE in which the factors were the different categories of vehicles and their levels were the number of them. As the reader might note, the combinatorial challenge make it impossible to run a full factorial design, which in this case it might imply to run at least 19683 different scenarios. For that reason, we implemented the Federov algorithm [14] which allowed the reduction of the amount of configurations to evaluate by doing and intelligent selection of them. After implementing this algorithm, the number of scenarios to evaluate was reduced to only to 27 as Table 5 presents. 
Table 5 Design of Experiments based on Federov's Algorithm

\begin{tabular}{|c|c|c|c|c|c|c|c|c|c|}
\hline $\begin{array}{l}\text { Scenario } \\
\text { Number }\end{array}$ & Loader & Bulk1 & Bus1 & Stairs1 & Stairs2 & $\begin{array}{r}\text { Water } \\
\text { service }\end{array}$ & Bulk2 & $\begin{array}{l}\text { Fuel } \\
\text { truck }\end{array}$ & Bus2 \\
\hline 219 & 1 & -1 & -1 & 1 & 1 & -1 & -1 & -1 & -1 \\
\hline 723 & 1 & -1 & 1 & 1 & 1 & 1 & -1 & -1 & -1 \\
\hline 4609 & -1 & -1 & 1 & 1 & 1 & -1 & -1 & 1 & -1 \\
\hline 4867 & -1 & 1 & -1 & -1 & -1 & 1 & -1 & 1 & -1 \\
\hline 4941 & 1 & 1 & 1 & 1 & -1 & 1 & -1 & 1 & -1 \\
\hline 5049 & 1 & 1 & 1 & -1 & 1 & 1 & -1 & 1 & -1 \\
\hline 5077 & -1 & -1 & -1 & 1 & 1 & 1 & -1 & 1 & -1 \\
\hline 5232 & 1 & -1 & 1 & 0 & 0 & -1 & 0 & 1 & -1 \\
\hline 5851 & -1 & -1 & 1 & -1 & -1 & -1 & 1 & 1 & -1 \\
\hline 5894 & 0 & 1 & -1 & 1 & -1 & -1 & 1 & 1 & -1 \\
\hline 5968 & -1 & -1 & -1 & 1 & 0 & -1 & 1 & 1 & -1 \\
\hline 6019 & -1 & 1 & 1 & -1 & 1 & -1 & 1 & 1 & -1 \\
\hline 8202 & 1 & -1 & 1 & -1 & 1 & -1 & 1 & -1 & 0 \\
\hline 12555 & 1 & 1 & 1 & 1 & 0 & -1 & 1 & 1 & 0 \\
\hline 13123 & -1 & -1 & -1 & -1 & -1 & -1 & -1 & -1 & 1 \\
\hline 13131 & 1 & 1 & -1 & -1 & -1 & -1 & -1 & -1 & 1 \\
\hline 13443 & 1 & 0 & 1 & 1 & -1 & 0 & -1 & -1 & 1 \\
\hline 13687 & -1 & 1 & 1 & 1 & -1 & 1 & -1 & -1 & 1 \\
\hline 14312 & 0 & -1 & -1 & 1 & 1 & 0 & 0 & -1 & 1 \\
\hline 14367 & 1 & -1 & -1 & 0 & -1 & 1 & 0 & -1 & 1 \\
\hline 15087 & 1 & -1 & 1 & -1 & -1 & 1 & 1 & -1 & 1 \\
\hline 15136 & -1 & 1 & 0 & 1 & -1 & 1 & 1 & -1 & 1 \\
\hline 15255 & 1 & 1 & 1 & -1 & 1 & 1 & 1 & -1 & 1 \\
\hline 17760 & 1 & -1 & 1 & -1 & -1 & 0 & -1 & 1 & 1 \\
\hline 18007 & -1 & 1 & 1 & -1 & -1 & 1 & -1 & 1 & 1 \\
\hline 19012 & -1 & 0 & -1 & 1 & -1 & -1 & 1 & 1 & 1 \\
\hline 19029 & 1 & -1 & 1 & 1 & -1 & -1 & 1 & 1 & 1 \\
\hline
\end{tabular}

Table 5 has been encoded for the different number of vehicles, it corresponds to -1 as 2 vehicles, 0 corresponds to 5 vehicles and +1 corresponds to 8 vehicles.

After running the 27 scenarios, we performed again the ANOVA for identifying which vehicles were the most influential for the objective pursued. In this case and due to the few amounts of points for the analysis it was not possible to consider the $2^{\text {nd }}$ order interactions. Therefore we could only make an analysis of the first order interactions or the direct effect of the use of vehicles.

Figure 9 presents a scatter plot that together with ANOVA helps identifying the influence in the TAT of some parameters which later would be used for improving the optimization search. 


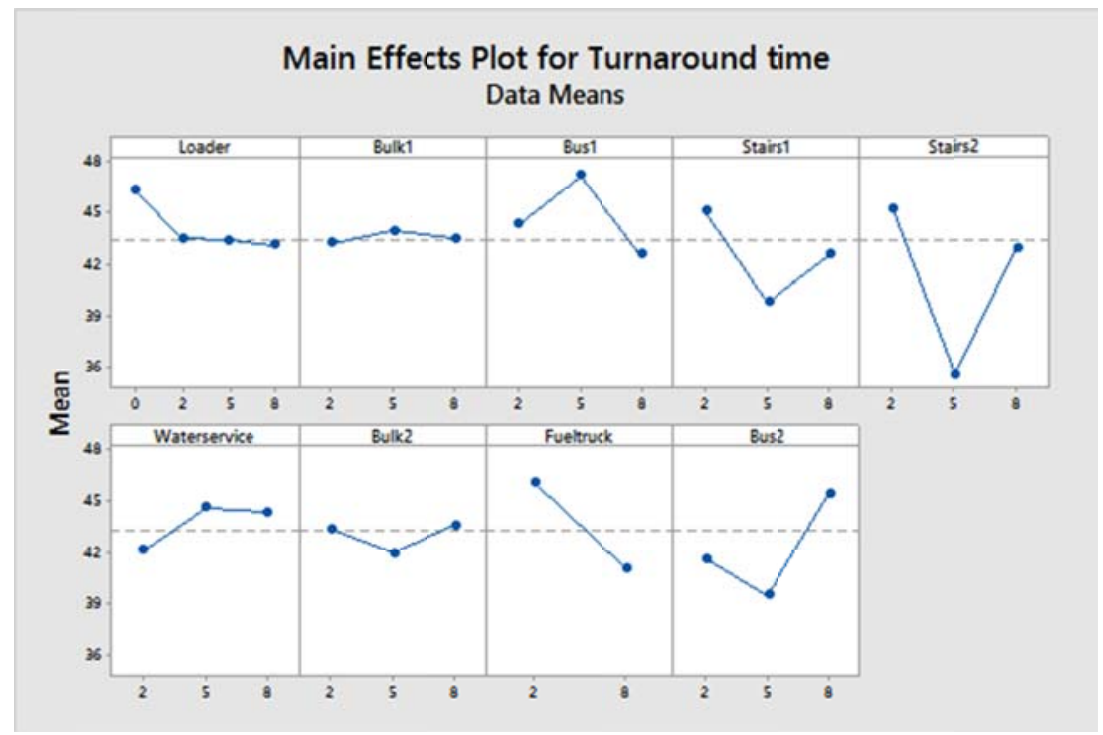

Fig. 9 Dependency of TAT on the modification of vehicle numbers

From the scatter plot we could identify that some values of vehicles minimize the turnaround time, namely Stairs 1, Stairs 2, Bulk2, fuel truck, Bus2. This result was also used for the last phase of the optimization.

In addition to this analysis, we performed the ANOVA for identifying which vehicles were the most influential for the TAT. We identified that the main factors that affect the turnaround time was firstly the fuel truck, and then the use of the stairs. Figure 10 illustrates the outcome of the ANOVA analysis. 


$\begin{array}{lrrrrr}\text { Analysis of Variance } & & & & \\ \text { Source } & \text { DF } & \text { Adj SS } & \text { Adj MS } & \text { F-Value } & \text { P-Value } \\ \text { Model } & 9 & 295.964 & 32.885 & 2.99 & 0.025 \\ \quad \text { Linear } & 9 & 295.964 & 32.885 & 2.99 & 0.025 \\ \quad \text { Loader } & 1 & 16.066 & 16.066 & 1.46 & 0.243 \\ \text { Bulk1 } & 1 & 0.078 & 0.078 & 0.01 & 0.934 \\ \text { Bus1 } & 1 & 2.848 & 2.848 & 0.26 & 0.617 \\ \text { Stairs1 } & 1 & 36.351 & 36.351 & 3.30 & 0.087 \\ \text { Stairs2 } & 1 & 36.745 & 36.745 & 3.34 & 0.085 \\ \text { Waterseryice } & 1 & 7.392 & 7.392 & 0.67 & 0.424 \\ \quad \text { Bulk2 } & 1 & 7.287 & 7.287 & 0.66 & 0.427 \\ \quad \text { Fueltruck } & 1 & 115.960 & 115.960 & 10.54 & 0.005 \\ \quad \text { Bus2 } & 1 & 3.275 & 3.275 & 0.30 & 0.592 \\ \text { Error } & 17 & 187.046 & 11.003 & & \\ \text { Total } & 26 m 48.0120 & & & \end{array}$

Fig.10 ANOVA for the $1^{\text {st }}$ order interaction in the TAT analysis

This result is very important, since it suggests that when someone is interested in improving the TAT of this particular airport, he should ensure that there are enough amounts of fuel trucks and stairs. The Pareto chart of standardized effects in turn can also illustrate the impact of the fuel truck and the stairs as the reader can see in Figure 11.

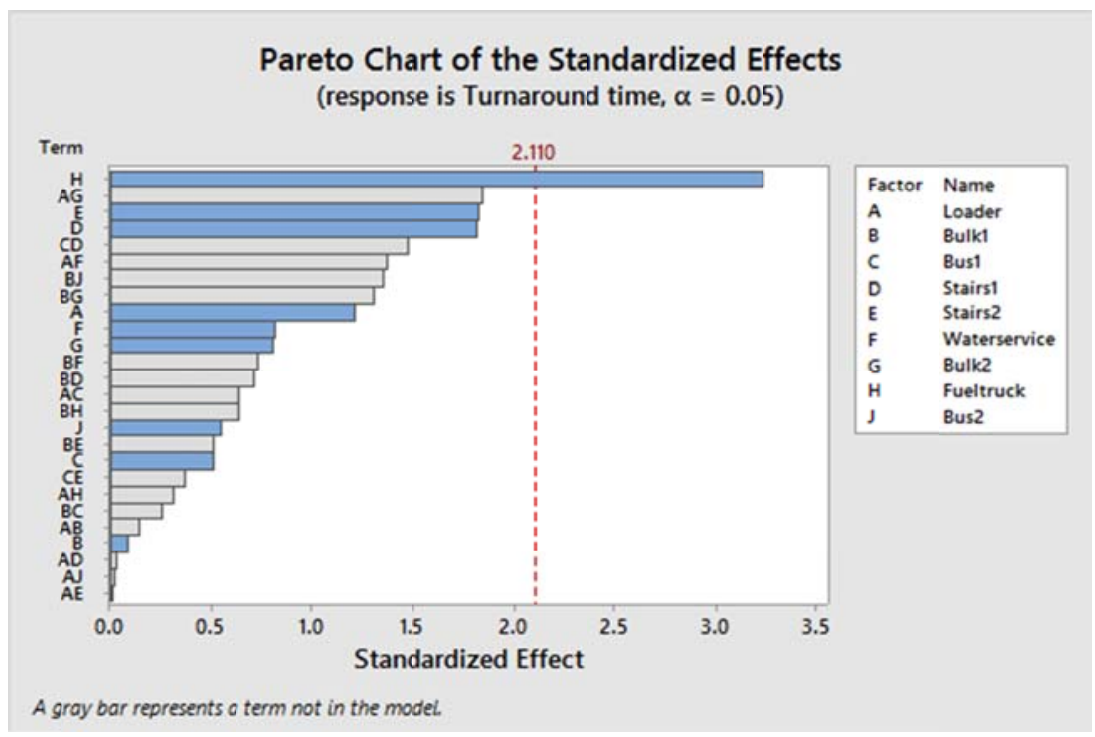

Fig. 11 Standardized effects for the TAT evaluation 


\subsection{Optimizing the model response}

In the next phase of the analysis, we used the previous results for making a more informed search over the solution space of the simulation model.

In most of the commercial simulation tools there are programs embedded that perform a simulation-based optimization. This optimization is performed by parameterizing the simulation model and then undergoing a search in the domain of the parameters' values. The search is done in most of the cases using a bruteforce approach in which the program just tests different values and make several replications of the model in order to find the best values for the objective function. As the reader might infer, the more parameters and the higher the range of the domain the more time consuming the search becomes. For this reason, it is necessary to support the search, otherwise the required time to get to a good solution could take a lot of time, and sometimes it would become unfeasible to wait for a solution.

For the previous reason, in the next stage, we used the information obtained from the previous analysis for restricting the domain of the search in the algorithm of the optimization program embedded in SIMIO.

\section{Optimization Phase}

The final phase of the methodology focuses on getting the optimal values for the Turnaround Time which is the factor analysed in this study.

OptQuest is an optimization tool present in SIMIO, and it allows the user to specify the objective function(s), domains, independent variables which will define the search space, and it will use the simulation model for performing the evaluation of the objective function. As the reader might know, the search over a high dimensional space takes from some minutes to even days, for that reason it is important to define wisely the boundaries and objectives of the optimizer.

For making the search as fast as possible, we implemented the so-called Restricted Search in which we fixed restrictions to the Optimizer for making the search under the boundaries we defined in the previous analysis. 
The restricted search was limited to the following domain of the vehicles:

- $\quad$ Fuel Truck: 8 vehicles (fixed value)

- Stairs1: 5 vehicles (fixed value)

- Stairs2: 5 vehicles (fixed value)

- Bulk2: 5 vehicles (fixed value)

- Bus2: 5 vehicles (fixed value)

- Loader: [2..8]

- Bus1: [2..8]

- Bulk1: [2..8]

- Water: [2..8]

The numbers assigned were taken from the insight obtained by the previous phase in which we could identify that the best performance could be achieved somewhere in the region near the fixed values of the initial five vehicles. For the remaining vehicles we relaxed the search so that the algorithm of the optimizer can search freely on the complete domain.

For the sake of comparison we also performed the same optimization but letting OptQuest make the search freely, for this reason we called it as Free Search.

The obtained results for both searches are presented in the following table.

Table 6 Analysis of the Optimized Search

\begin{tabular}{|c|c|c|c|c|c|c|}
\hline \multirow{2}{*}{\multicolumn{2}{|c|}{$\begin{array}{l}\text { Type of Search } \\
\text { Maximum number of combinations }\end{array}$}} & \multicolumn{3}{|c|}{ Free Search } & \multicolumn{2}{|c|}{ Restricted Search } \\
\hline & & 50 & 100 & 300 & 50 & 100 \\
\hline \multirow{3}{*}{$\begin{array}{l}\text { Solution with } \\
\text { minimum TAT }\end{array}$} & Turnaround Time (min) & 29.41 & 29.23 & 29.40 & 29.56 & 29.56 \\
\hline & Number of Vehicles & 65 & 67 & 58 & 56 & 56 \\
\hline & Number of combinations & 17 & 37 & 204 & 5 & 5 \\
\hline \multirow{3}{*}{$\begin{array}{l}\text { Solution with } \\
\text { minimum number } \\
\text { of vehicles }\end{array}$} & Turnaround time (min) & 42.46 & 42.46 & 42.46 & 37.01 & 29.56 \\
\hline & Number of Vehicles & 18 & 18 & 18 & 36 & 56 \\
\hline & Number of Combinations & 2 & 2 & 2 & 2 & 5 \\
\hline
\end{tabular}

For limiting the speed of calculation and time to get the results it is also necessary to establish some limits for the allowed number of combinations for providing the solution. In our example, for making a comparison between the free and the restricted search, we set the limits to 50 and 100 . In addition we also set another limit for the free search just for having an idea of the improvement that can be achieved if the analyst had enough time to let the model run.

\section{Maximum combinations 50}

Regarding the performance of the approach, when we pay attention to the scenarios, the first one is the limited by 50 permutations. 
Table 6 illustrates that in terms of Turnaround time, the free search provides a slightly better solution than the restricted one, however the restricted search finds a similar solution with only 5 combinations and a smaller number of vehicles than the free search (56 compared to 65).

When we check the solution with the minimum number of vehicles, we identified that after two permutations the free search provides a solution with 18 vehicles and a turnaround time of $42.46 \mathrm{~min}$, while the restricted one provides a solution of 36 vehicles but with a shorter turnaround time of 37.01 minutes. These results supports the premise that limiting the search space based on the results of the DOE \& ANOVA it will provide a better starting point for the search.

\section{Maximum combinations 100}

Regarding the turnaround time, for this amount of maximum number of permutations we can appreciate that the achieved Turnaround times are very similar, however the restricted search finds a solution which is less costly since it uses only 56 vehicles while the free search 67 . In addition, the restricted search finds it with a minimum amount of permutations.

If we wanted to pay attention to a solution of minimum vehicles, the free search finds a suitable solution of 18 vehicles while the restricted one finds one of 36 vehicles but with a better turnaround time in the same amount of permutations which is in line with the previous example.

Regarding the free search with a limit of 300 permutations, we can appreciate that the results are not necessarily better, they can be even worse that a more restricted search. This can be noted in the turnaround time when we let it make a free search on a more relaxed fashion. This result also indicates the complexity of the solution space of this system.

\section{Conclusions}

Managing an airport system is a complex task in which the decision involves many variables, thus the decision makers require decision-support tools that provide them insight of the consequences of taking particular decisions.

In this work we presented a case of the analysis of an airport in the Netherlands which is currently under construction. For the decision makers it is important to identify what the most influential variables are in order to improve the performance. This is key for them since the more efficient the airport, the most attractive for airlines to move there.

In this work we illustrated how a structured methodology can help identifying the most influential decision variables for the system in place. With the identification of them, it is possible to use simulation together with optimization for finding the values of the decision variables that improve the performance of the airport under study; in this case we put focus on the turnaround time. The results illustrate that certainly the methodology successfully drives the search 
space into a region of good solutions so we could obtain very good values without performing a time-consuming search.

The methodology has been implemented in the case of an integral airport model developed in SIMIO using OptQuest as the optimization tool. However this methodology can be easily implemented in a different area using a different simulation tool and a different optimizer.

\section{References}

1. ACI,2014,<https:/www.acieurope.org/component/content/article/29article/103-world-airport-traffic-data.html>

2. Arias, P., Guimarans D., Boosten G., Mujica M., 2013. A methodology combining optimization and simulation for real applications of the Stochastic Aircraft Recovery Problem'. In Proc. of the EUROSIM'13 , Cardiff, U.K.

3. Balakrishnan H., Chandran B., 2010. Algorithms for scheduling runway operations under constrained position shifting. Operations Research, 58 (6), pp.1650-1665

4. Beasley J.E., Krishnamoorthy M., Sharaiha Y.M., Abramson D., 2000. Scheduling aircraft landings-the static case. Transportation Science, pp.180197

5. Beasley J.E., Krishnamoorthy M., SharaihaY.M., Abramson D, 2004. Displacement problem and dynamically scheduling aircraft landings'. Journal of the operational research society vol.55, pp.54-64

6. Beasley J.E., Sonander J., Havelock P., 2001. Scheduling aircraft landings at London heathrow using a population heuristic. Journal of the Operational Research Society, vol.52, pp.483-493

7. Bolat A., 2000. Procedures for providing robust gate assignments for arriving aircrafts. European Journal of Operational Research, 120 (1), pp.63-80

8. DeNeufville, R., Scholtes, S., 2011. Flexibility in Engineering Design. MIT Press

9. Dorndorf U., Drexlb A., Nikulin Y., Peschc E., 2007. Flight gate scheduling: State-of-the-art and recent developments. Omega, 35 (3), p.326-334

10. Eurocontrol, 2013, “Challenges of Growth 2013”, Technical report

11. Eurocontrol, December 2016. Coda Report: All-causes Delay to Air Transport in Europe. Technical Report

12. Eurocontrol,2015. Industry Monitor, Issue N 174. 01/07/2015

13. $\mathrm{Hu}$ X., Chen W., 2005. Receding Horizon Control for Aircraft Arrival Sequencing and Scheduling. IEEE Transactions On Intelligent Transportation Systems, vol.6, no.2

14. Federov V.V. 1972.Theory of Optimal Experiments. Eds W. J. Studden and E. M. Klimko. New York, Academic Press

15. Jimenez E., 2014. Airport strategic planning in the context of low-cost carriers ascendency: insights from the European experience. $\mathrm{PhD}$ thesis, Universidad do Porto 
16. Khadilkar H., Balakrishnan H., 2014. Network Congestion Control of Airport Surface Operations. AIAA Journal of Guidance, Control and Dynamics, 7 (3), pp.933-940

17. Longo F., 2013. On the short period production planning in Industrial Plants: A real Case Study. International Journal of Simulation \& Process Modelling, vol.8, pp.17-28

18. Michelin A., Idan M., Speyer J.L. 2009. Merging of air traffic flows. AIAA Guidance, Navigation, and Control Conference 10-13, Chicago, Illinois

19. Mujica M., 2015. Check-In allocation improvements through the use of a Simulation-Optimization Approach. Transportation Research Part A, pp.320335

20. Mujica M., de Bock N., Boosten G., Jimenez E., Pinho J., 2015. SimulationBased Turnaround Evaluation for Lelystad Airport. Air Transport Research Society World Conference. July 2-5, Singapore

21. Murca M.C.R., Müller C., 2015. Control-based optimization approach for aircraft scheduling in a terminal area with alternative arrival routes. Transportation Research Part E, vol.73, pp.96-113

22. Narciso M.E., Piera M.A., 2015. Robust gate assignment procedures from an airport management perspective. Omega, 50, pp.82-95

23. OptQuest webpage < http://www.opttek.com/products/optquest/>

24. Pujet N., Delcaire B., Feron E., 1999. Input-output modeling and control of the departure process of congested airports. A collection of technical papers: AIAA Guidance, Navigation and Control Conference and Exhibit, Part 3, pp.1835-1852. August 9-11, Portland (Oregon, USA)

25. Scala P., Mujica M., Zuniga C.A., 2015. Assessing the future TMA Capacity of Lelystad Airport using Simulation. Air Transport and Operations Symposium. July 20-22, Delft (The Netherlands)

26. Scala P., Mujica M., De Bock N., 2015. Modular Approach For Modelling An Airport System. EMSS European Modeling \& Simulation Symposium. September 21-23, Bergeggi (Italy)

27. SchipholMagazine,2014 <http://trafficreview2014.schipholmagazines.nl/airtransportmovements.html\#atmmainairlinesa>

28. Schiphol Group, 2014. Ondernemingsplan Lelystad Airport. March 2014

29. Schiphol Group, 2016 .<http://www.schiphol.nl/SchipholGroup1/ Onderneming/Statistieken/VerkeerVervoerCijfers1.html>

30. Simaiakis I., Balakrishnan H., 2014. A Queuing Model of the Airport Departure Process. Transportation Science, accepted, July 2014

31. SIMIO web page <http:www.simio.com>

32. van Baren, G. and Treve, V. 2015. The current practice of separation delivery at major european airports.

$<$ http://www.atmseminar.org/seminarContent/seminar11/ papers/466Van\%20Baren_0126150311-Final-Paper-5-7-15.pdf.>

33. Zuniga C.A., Delahaye D., Piera M.A., 2011. Integrating and Sequencing Flows in Terminal Maneuvering Area by Evolutionary Algorithms. , DASC 
2011, 30th IEEE/AIAA Digital Avionics Systems Conference, Seattle, United States

34. Zuniga C.A., Piera M.A., Ruiz S., Del Pozo I., 2013. A CD \&CR causal model based on path shortening/path stretching techniques. Transportation Research Part C, 33, pp.238-256 